\title{
Correlation Statistics of the $\alpha-\mu$ Fading Channels
}

\author{
Ugo Silva Dias and Michel Daoud Yacoub
}

\begin{abstract}
Resumo-Medições de campo em ambientes indoor e outdoor são utilizadas para validar a função de autocorrelação obtida de maneira exata para o modelo de desvanecimento $\alpha-\mu$. Além disso, aproximações precisas e em forma fechada são obtidas e também validadas para a função de autocorrelação e para o espectro de potência da envoltória $\alpha-\mu$. São realizadas comparações e um excelente ajuste às medidas de campo é observado em ambos os ambientes isotrópicos e anisotrópicos.
\end{abstract}

Palavras-Chave-Distribuição $\alpha-\mu$, autocorrelação, medições de campo, espectro de potência.

Abstract-Indoor and outdoor field trial measurements are used to validate the autocorrelation function derived in an exact manner for the $\alpha-\mu$ fading signal. In addition, accurate closedform approximations for both autocorrelation function and to the power spectrum of the $\alpha-\mu$ envelope are obtained and also validated. Comparisons are performed and an excellent fit to the field measurements is found in both isotropic and anisotropic environments.

Keywords $-\alpha-\mu$ distribution, autocorrelation, field trials, power spectrum.

\section{INTRODUCTION}

In wireless communications, the multipath fading phenomenon has been characterized by several important statistics models, notably Rayleigh, Rice, Hoyt (Nakagami- $q$ ), Nakagami$m$, and Weibull [1]-[3]. Recently [4], a general physical fading model, namely the $\alpha-\mu$ model, has been described that considers a signal composed of clusters of multipath waves propagating in a nonhomogeneous environment. The resultant envelope in such an environment then follows the $\alpha-\mu$ distribution, which includes as special cases Nakagami- $m$ and Weibull. Its flexibility renders it adaptable to situations in which neither of these two distributions yield good fit [4]. Experimental data supporting the usefulness of the Nakagami$m$ and Weibull fading models have been widely reported in the literature (e.g., [5]-[8]). However, to the best of the authors' knowledge, works depicting practical situations in which higher-order statistics of $\alpha-\mu$ distribution are used have not been reported in the literature.

In this paper, some second-order statistics for the $\alpha-\mu$ fading channels are (i) derived, (ii) validated through field measurements, and (iii) compared with those of its particular cases. With such a target, the autocorrelation function is obtained in an exact manner. In addition, experimental data are collected that fit both isotropic and anisotropic conditions. As noted by several authors (e.g. [9]), the assumption of a uniform probability density function (PDF) for the angle of arrival

The authors are with the Wireless Technology Laboratory (WissTek), Department of Communications, School of Electrical and Computer Engineering, University of Campinas, PO Box 6101, 13083-852, Campinas, SP, Brazil (email: [ugo,michel]@wisstek.org). This work was partly supported by FAPESP (06/06825-8)
(AOA) may introduce errors on the second-order statistics, particularly correlation functions and level crossing rates. In addition, a very simple, accurate closed-form approximation to the autocorrelation function is obtained. This is then used to derive accurate closed-form approximation to the power spectrum of the $\alpha-\mu$ envelope, which is also validated through field measurements.

\section{THE $\alpha-\mu$ AUTOCORRELATION FUnCtion}

The temporal autocorrelation function (ACF) $A_{R}(\tau)$ of the $\alpha-\mu$ envelope $R$ can been obtained from its generalized joint moments, as derived in [4, Eq. 29], as

$$
\begin{aligned}
& A_{R}(\tau) \triangleq E[R(\tau) R(t+\tau)]= \\
& \frac{\hat{r}^{2} \Gamma^{2}\left(\mu+\frac{1}{\alpha}\right)_{2} F_{1}\left(-\frac{1}{\alpha},-\frac{1}{\alpha} ; \mu ; \rho(\tau)\right)}{\mu^{\frac{2}{\alpha}} \Gamma^{2}(\mu)}
\end{aligned}
$$

where $\hat{r}=\sqrt[\alpha]{E\left[R^{\alpha}\right]}$ is the $\alpha$-root mean value of $R^{\alpha}, \Gamma(z)=$ $\int_{0}^{\infty} t^{z-1} \exp (-t) d t$ is the Gamma function, ${ }_{2} F_{1}(\cdot, \cdot ; \cdot ; \cdot)$ is the hypergeometric function [10, Eq. 15.1.1], $\rho(\tau)$ is the temporal autocorrelation coefficient (ACC) of a squared Rayleigh envelope [4, Eq. 36], and $E[\cdot]$ denotes the expectation operator. Using the space-time duality of the wireless channel [11], then $\omega_{D} \tau=2 \pi d / \lambda$, where $\omega_{D}$ is the maximum Doppler frequency in $\mathrm{rad} / \mathrm{s}, d$ denotes distance, and $\lambda$ is the carrier wavelength. Thus, the spatial ACF $A_{R}(d)$ of $\mathrm{R}$ can be straightforwardly found in terms of the spacial ACC $\rho(d)$ as

$$
A_{R}(d)=\frac{\hat{r}^{2} \Gamma^{2}\left(\mu+\frac{1}{\alpha}\right){ }_{2} F_{1}\left(-\frac{1}{\alpha},-\frac{1}{\alpha} ; \mu ; \rho(d)\right)}{\mu^{\frac{2}{\alpha}} \Gamma^{2}(\mu)}
$$

In this work, we address the general case of anisotropic/isotropic fading scenarios, for which the distribution of the angle of arrival of the multipath waves are nonuniform/uniform. A widely used model for the directional AOA is the parametric Von Mises (Tikhunov) distribution [12]. For this model, the spatial squared Rayleigh ACC can be obtained as (3), as shown at the top of the next page, in which $0 \leq \zeta \leq 1$ indicates the amount of directional reception, $I_{0}(\cdot)$ is the modified Bessel function of the first kind and zeroth order [10, Eq. 9.6.16], $\varphi \in[-\pi, \pi)$ represents the mean direction of the AOA, and $k \geq 0$ controls the beamwidth. In particular, for $\zeta=0$, the composite anisotropic/isotropic model reduces to the isotropic scenario with uniform AOA, for which $\rho(d)=J_{0}^{2}(2 \pi d / \lambda)$, where $J_{0}(\cdot)$ is the Bessel function of the first kind and zeroth order [13, Eq. 8.401].

\section{A. The Moment-based $\alpha$ - $\mu$-estimator}

The moments of the $\alpha-\mu$ envelope are given as [4] $E\left[R^{k}\right]=$ $\frac{\hat{r}^{k} \Gamma(\mu+k / \alpha)}{\mu^{k / \alpha} \Gamma(\mu)}$. From this, an equality is defined that is useful for 


$$
\rho(d)=\left|\frac{\zeta I_{0}\left(\sqrt{k^{2}-(2 \pi d / \lambda)^{2}+j 4 \pi k d \cos (\varphi) / \lambda}\right)+(1-\zeta) J_{0}(2 \pi d / \lambda) I_{0}(k)}{I_{0}(k)}\right|^{2}
$$

the estimation of the parameters of this distribution. In essence [4],

$$
\frac{E^{2}\left[R^{\beta}\right]}{E\left[R^{2 \beta}\right]-E^{2}\left[R^{\beta}\right]}=\frac{\Gamma^{2}(\mu+\beta / \alpha)}{\Gamma(\mu) \Gamma(\mu+2 \beta / \alpha)-\Gamma^{2}(\mu+\beta / \alpha)}
$$

in which $\beta$ is chosen arbitrarily. For two distinct and arbitrary values of $\beta$, two equations are set up so that the physical parameters $\alpha$ and $\mu$ are encountered. For a particular case in which $\beta=1$ and $\beta=2$, (4) yields an estimator in terms of the first and second moments. Of course, from (4), other moment-based estimators can be used.

\section{B. The Nonlinear $\zeta-\varphi$ - $k$-estimator}

The unknown parameters $\zeta, \varphi$, and $k$ of the nonlinear model (3) can be estimated by means of a numerical procedure as follows. They can be evaluated by the nonlinear least squares method [14], which provides consistent estimates. In essence,

$$
(\hat{\zeta}, \hat{\varphi}, \hat{k})=\underset{\zeta, \varphi, k}{\operatorname{argmin}} M S E
$$

where $M S E=N^{-1} \sum_{l=1}^{N}[\hat{\rho}(d)-\rho(d)]^{2}$ with $\rho(d)$ given in (3) and $\mathrm{N}$ is the total number of sample data. In particular, for the isotropic scenario with uniform AOA, $M S E_{\text {isotropic }}=$ $N^{-1} \sum_{l=1}^{N}\left[\hat{\rho}(d)-J_{0}^{2}(2 \pi d / \lambda)\right]^{2}$.

\section{The $\alpha-\mu$ EnVelope Power Spectrum}

The power spectrum $S_{R}(\gamma)$ of the $\alpha-\mu$ fading envelope $R$ is the Fourier transform ${ }^{1}$ of its ACF $A_{R}(d)$ as defined in (2). Although this leads to an exact calculation, it seems that no closed-form expression can be found. In this section, a simple, accurate closed-form approximation to $S_{R}(\gamma)$ is derived for the isotropic scenario. To this end, the following expansion of the hypergeometric function ${ }_{2} F_{1}(\cdot)$ is used [10]

$$
\begin{array}{r}
{ }_{2} F_{1}\left(-\frac{1}{\alpha},-\frac{1}{\alpha} ; \mu ; \rho(d)\right)=1+\frac{\rho(d)}{\alpha^{2} \mu}+\frac{(1-\alpha)^{2} \rho^{2}(d)}{2 \alpha^{4}\left(\mu^{2}+\mu\right)}+ \\
+\frac{(1-2 \alpha)^{2}(1-\alpha)^{2} \rho^{3}(d)}{6 \alpha^{6}\left(\mu^{3}+3 \mu^{2}+2 \mu\right)}+\ldots
\end{array}
$$

In (6), dropping the terms of order beyond two, the exact $\alpha-\mu$ ACF $A_{R}(d)$ can be approximated by $\tilde{A}_{R}(d)$ as

$$
A_{R}(d) \approx \tilde{A}_{R}(d)=\frac{\hat{r}^{2} \Gamma^{2}\left(\mu+\frac{1}{\alpha}\right)}{\mu^{\frac{2}{\alpha}} \Gamma^{2}(\mu)}\left(1+\frac{\rho(d)}{\alpha^{2} \mu}\right)
$$

The maximum deviation between the exact (2) and the approximate (7) solutions occurs for $d=0$, for the hypergeometric series in this case contains only positive terms

${ }^{1}$ The Fourier transform $\mathcal{F}(\gamma)$ of a function $f(x)$ is defined here as $\mathcal{F}(\gamma)=$ $\int_{-\infty}^{\infty} f(x) \exp (-j \gamma x) d x$.

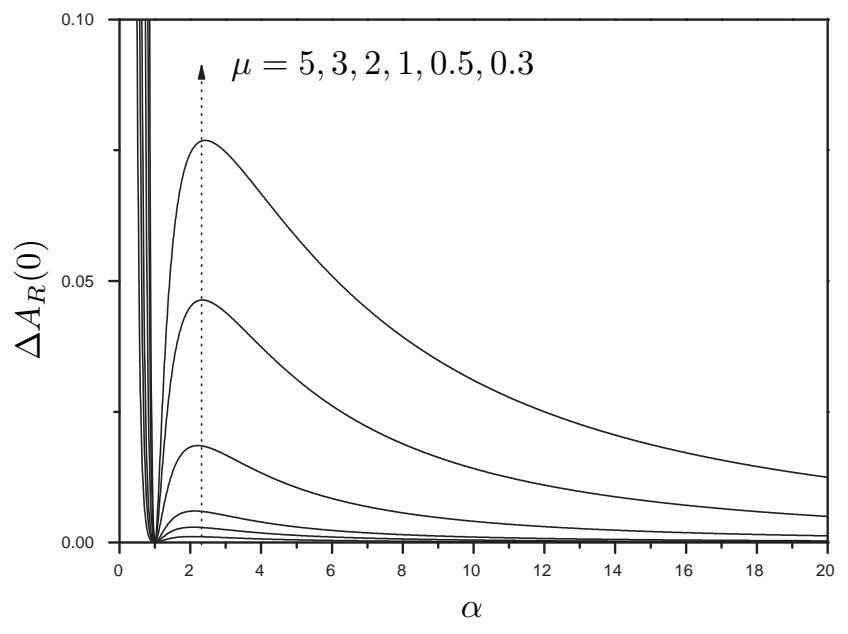

Fig. 1. Maximum deviation of the approximated and exact $\alpha-\mu$ autocorrelation functions.

and $\rho(d)$ has its maximum at $d=0$. Defining $\Delta A_{R}(0)=$ $\left[A_{R}(0)-\tilde{A}_{R}(0)\right] / \hat{r}^{2}$, Figs. 1 and 2 plot this deviation as a function of $\alpha$ and $\mu$. Indeed, the deviation is null for $\alpha=1$ independently of $\mu$ values, i.e. the approximate formulation in (7) is indeed exact for $\alpha=1$. In addition, for $\alpha>1$ and $\mu \geq 1$, such a deviation is smaller than $1.85 \%$. As $\alpha \rightarrow \infty$ and/or $\mu \rightarrow \infty$ both $A_{R}$ and $\tilde{A}_{R}$ tend to $\hat{r}^{2}$ and $\Delta A_{R}(0)=0$. For $\alpha \rightarrow 0$ and/or $\mu \rightarrow 0$, the deviation tends to infinity. However, $\alpha<1$ and $\mu<0.5$ are rarely found in real situations. In fact, $\alpha<1$ and $\mu<0.5$ lead to a condition corresponding to a fading parameter (Nakagami- $m$ parameter) smaller than 0.09375 . Moreover, $\alpha>1$ and $\mu \geq 1$ correspond to a fading parameter greater than 0.25 . Thus, for practical purposes, the proposed approximation is indeed excellent.

Now, taking the Fourier transform of (7), as shall be seen, an accurate approximation to $S_{R}(\gamma)$ can be written in a closedform formula as

$$
\begin{aligned}
S_{R}(\gamma) \approx & \frac{\hat{r}^{2} \Gamma^{2}\left(\mu+\frac{1}{\alpha}\right)}{\mu^{\frac{2}{\alpha}} \Gamma^{2}(\mu)} \\
& \times\left[\delta(\gamma)+\frac{\lambda}{\pi^{2} \alpha^{2} \mu} K\left(\sqrt{1-\left(\frac{\lambda \gamma}{2}\right)^{2}}\right)\right]
\end{aligned}
$$

for $|\gamma|<2 / \lambda$, where $\delta(\cdot)$ is the Dirac delta function and $K(\cdot)$ is the complete elliptic integral of the first kind [7, Eq. 8.112.1]. As a check for the correctness of these results, we note that, for $\alpha=2$ (Nakagami- $m$ condition), $\mu=1$ (Weibull condition), and $(\alpha, \mu)=(2,1)$ (Rayleigh condition), (7) and (8) specialize into [5, Eqs. 4 and 5], [3, Eqs. 5 and 6], and [11, Eqs. 1.3-16 and 1.3-27], respectively. 


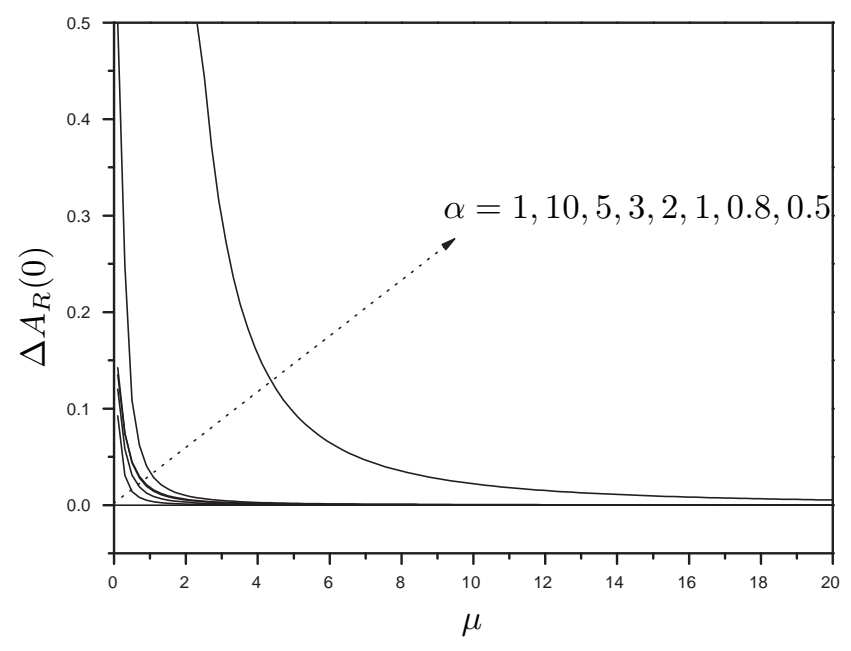

Fig. 2. Maximum deviation of the approximated and exact $\alpha-\mu$ autocorrelation functions.

\section{A. Sample Examples}

The exact and approximate normalized autocorrelation functions of the $\alpha-\mu$ envelopes are shown in Figs. 3 to 6, for isotropic $(\zeta=0)$ and anisotropic $(\zeta=0.8)$ fading scenarios, and for several different values of fading parameters. For the isotropic fading scenarios, $k=1$ and $\varphi=0$. Note the excellent match in all of the cases. The slightly poorer results for $(\alpha, \mu)<(1,0.5)$ are expected, because (7) deteriorates in this range, as already mentioned. Note that, as $\alpha$ and/or $\mu$ tend to infinity, the $\alpha-\mu$ process becomes constant functions, i.e., $A_{R}(d) \rightarrow \hat{r}$.

The approximation (8) to the $\alpha-\mu$ envelope power spectrum is compared to the exact formulation (obtained by numerical integration) in Figs. 7 and 8. Both exact and approximated spectra are plotted for $\mu=0.5,1$, and 3 and $\alpha=0.5,1$, and 2. (The DC component was omitted in these comparisons.) It can be seen that the differences are seen to be minimal for $(\alpha, \mu)>(1,0.5)$. The counterpart of the unity autocorrelation function as $\alpha$ and/or $\mu$ tend to infinity is purely DC spectrum, i.e., $S_{R}(\gamma) \rightarrow \hat{r} \delta(\gamma)$ for $\alpha \rightarrow \infty$ and/or $\mu \rightarrow \infty$.

\section{Field Trials and VAlidation}

A series of field trials was conducted at the University of Campinas (Unicamp), Brazil, in order to validate the autocorrelation function and the power spectrum of the $\alpha$ $\mu$ envelope. To this end, the transmitter was placed on the rooftop of one of the buildings and the receiver travelled through the campus as well as within the buildings. The mobile reception equipment was especially assembled for this purpose. Basically, the setup consisted of a vertically polarized omnidirectional receiving antenna, a low noise amplifier, a spectrum analyzer, data acquisition apparatus, a notebook computer, and a distance transducer for carrying out the signal sampling. The transmission consisted of a $\mathrm{CW}$ tone at 1.8 $\mathrm{GHz}$. The spectrum analyzer was set to zero span and centered at the desired frequency, and its video output used as the input of the data acquisition equipment. The local mean was

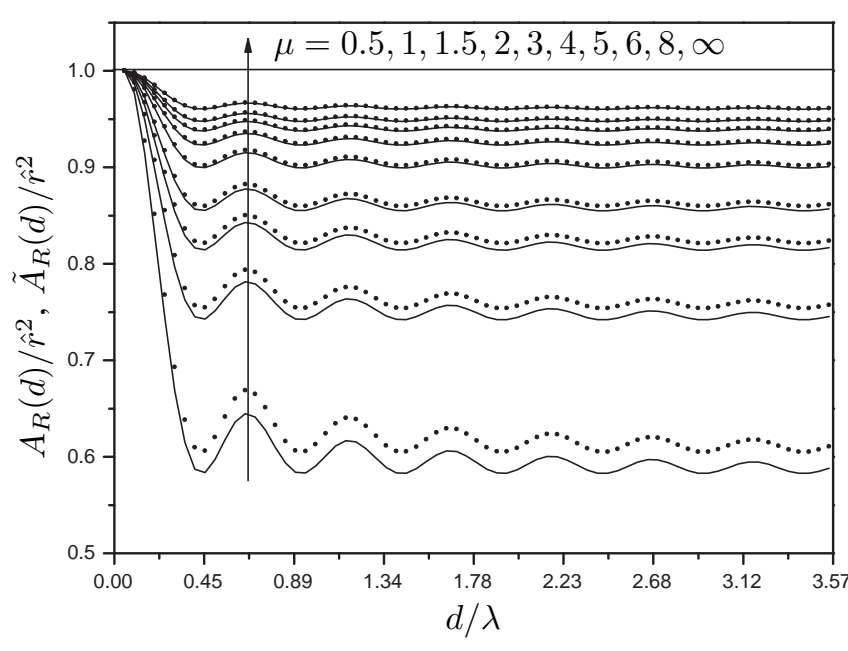

Fig. 3. $\alpha-\mu$ autocorrelation functions for isotropic fading scenarios, $\zeta=0$, and $\alpha=7 / 4$ (exact: solid; approximated: dot).

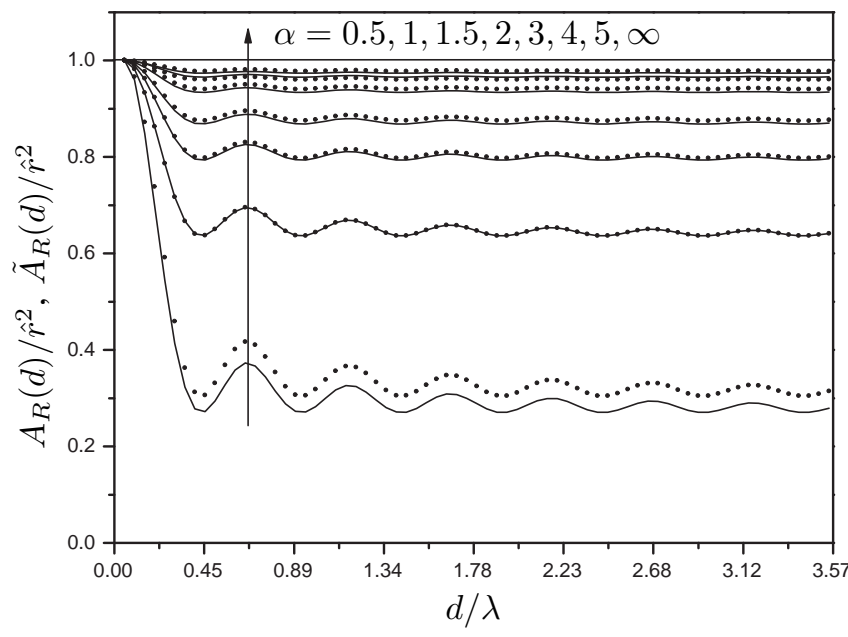

Fig. 4. $\alpha-\mu$ autocorrelation functions for isotropic fading scenarios, $\zeta=0$, and $\mu=7 / 4$ (exact: solid; approximated:dot).

estimated by the moving average method, with the average being conveniently taken over samples symmetrically adjacent to every point. From the data collected, the long term fading was filtered out and the fading parameters $\alpha$ and $\mu$, as defined previously, was estimated, as well as the anisotropic ones $\zeta$, $\varphi$, and $k$.

The normalized empirical autocorrelation was computed according to

$$
\widehat{A}_{R}(\Delta)=\frac{\sum_{i=1}^{N-\Delta} r_{i} r_{i+\Delta}}{\sum_{i=1}^{N-\Delta} r_{i}^{2}}
$$

where $r_{i}$ is the $i$-th sample of the amplitude sequence, $N$ is the total number of samples, $\Delta$ is the discrete relative distance difference, and $\widehat{A}_{R}($.$) denotes an empirical estimate of A_{R}($.$) .$ The empirical correlation coefficient was calculated as

$$
\widehat{\rho}(\Delta)=\frac{\widehat{A}_{R}(\Delta)-\widehat{E}^{2}(R)}{\widehat{V}\left(R^{2}\right)}
$$




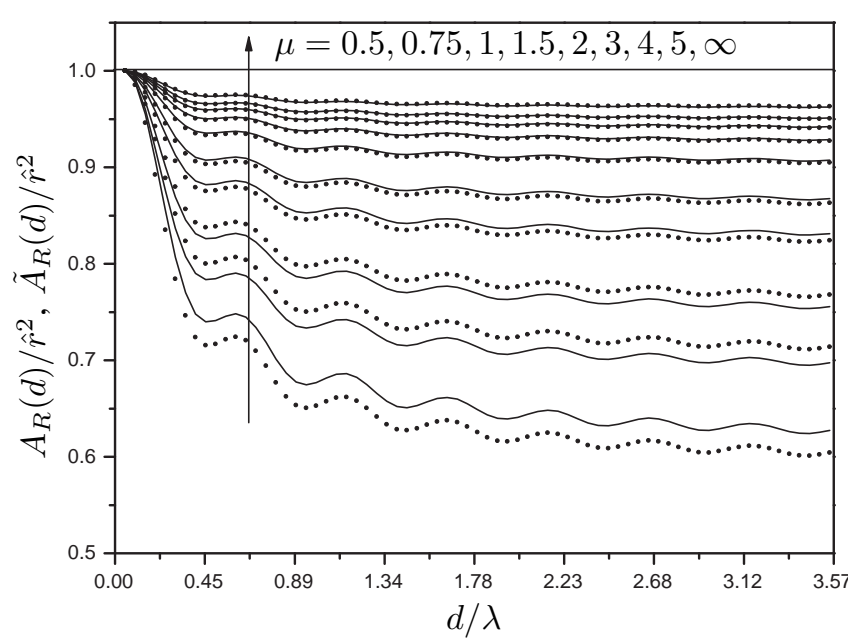

Fig. 5. $\alpha-\mu$ autocorrelation functions for anisotropic fading scenarios, $\zeta=$ $0.8, k=1, \varphi=0$, and $\alpha=7 / 4$ (exact: solid; approximated: dot).

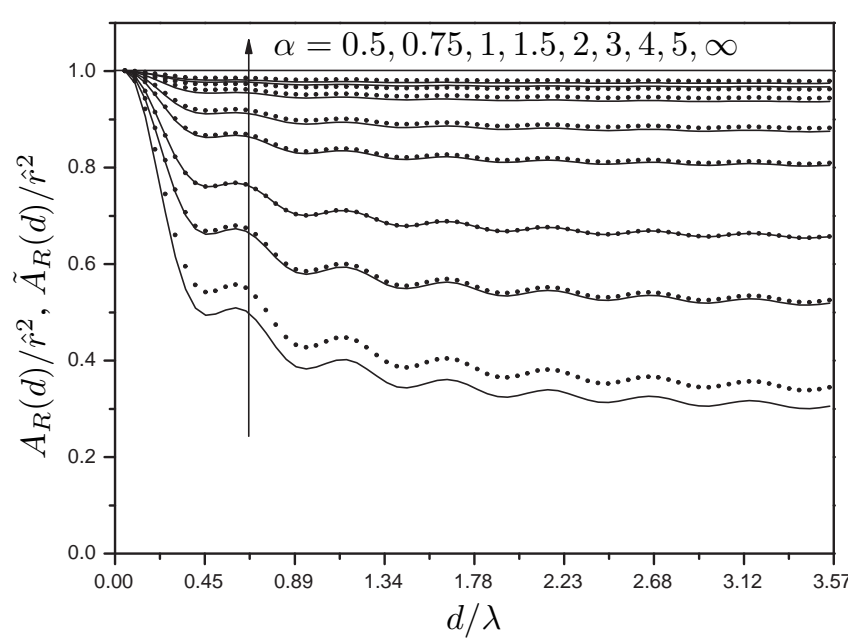

Fig. 6. $\alpha-\mu$ autocorrelation functions for anisotropic fading scenarios, $\zeta=$ $0.8, k=1, \varphi=0$, and $\mu=7 / 4$ (exact: solid; approximated: dot).

where $\widehat{E}(\cdot)$ and $\widehat{V}(\cdot)$ denote the estimate of the mean and the variance, respectively. Finally, the fading and anisotropic parameters could be estimated for all measurements.

The empirical autocorrelation function was compared against the corresponding theoretical formula (2) and plotted as a function of $d / \lambda$ with the same parameters $\alpha$ and $\mu$ estimated from the experimental data. Furthermore, the mean error deviation $^{2}, \epsilon$, was computed for each case. Figs. 9(a) to 9(c) show some sample plots comparing the experimental and theoretical anisotropic autocorrelation data for different values of the $\alpha-\mu$, Weibull, and Nakagami- $m$ distributions, as well as, for distinct parameters of the Von-mises model $(\zeta, \varphi$, and $k$ ). Table I shows the values of the parameters, the minimum

\footnotetext{
${ }^{2}$ The mean error deviation between the measured data $x_{i}$ and the theoretical value $y_{i}$ is defined as $\epsilon=\frac{1}{N} \sum_{i=1}^{N} \frac{\left|y_{i}-x_{i}\right|}{x_{i}}$, where $N$ is the number of points. For the present calculations, the errors were estimated for points in the interval $[0,1.43 \lambda]$, within which larger deviations occur.
}

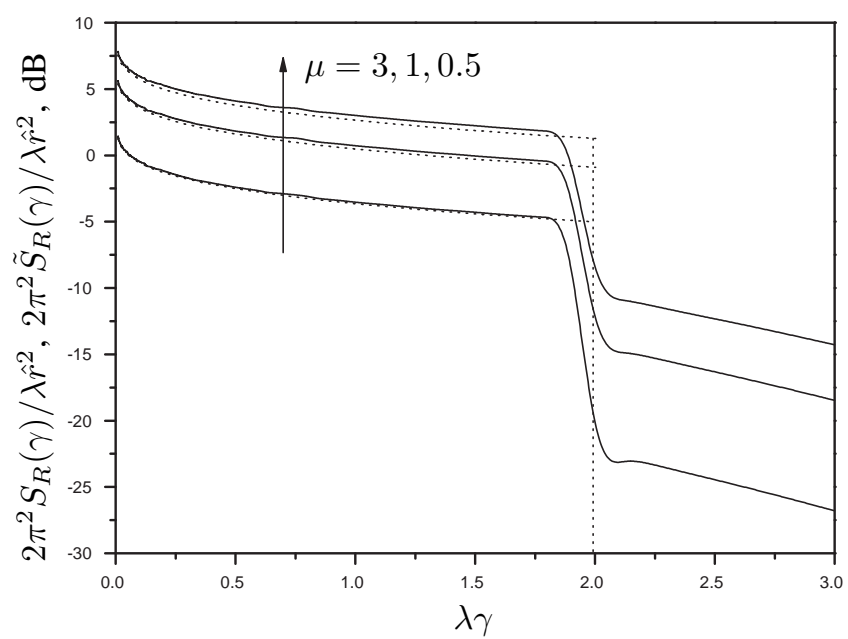

Fig. 7. $\alpha-\mu$ envelope power spectrum for isotropic fading scenarios and $\alpha=7 / 4$ (exact: solid; approximated: dot).

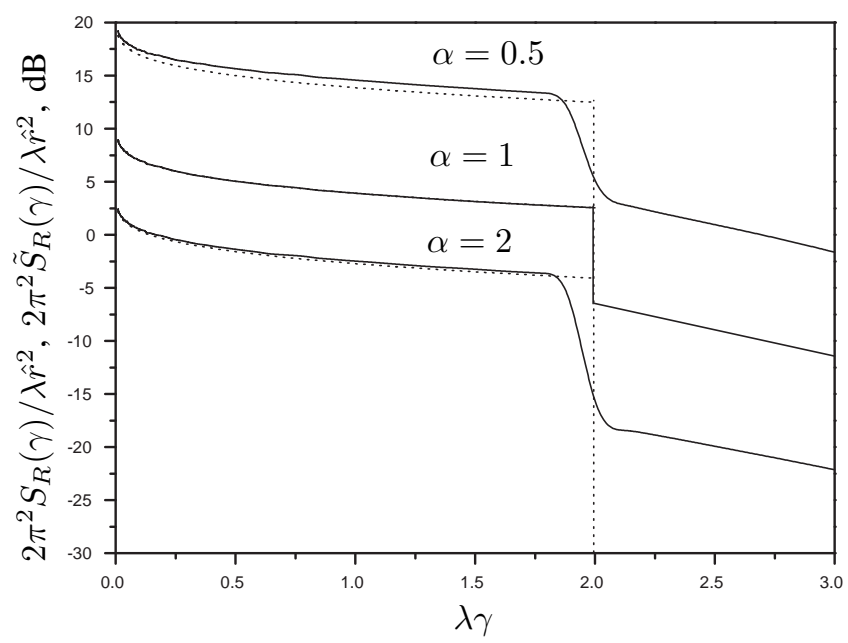

Fig. 8. $\alpha-\mu$ envelope power spectrum for isotropic fading scenarios and $\mu=7 / 4$ (exact: solid; approximated: dot).

MSE, and the mean error deviation $\epsilon$ for each sample result. Observe the excellent fit and how the theoretical curves tend to keep track of the changes of the concavity of the empirical data. The $\alpha-\mu$ model fits betters than Weibull and Nakagami$m$ ones, as expected, because it provides one more degree of freedom.

The $\alpha-\mu$ envelope power spectrum formulation (8) is then compared against that obtained empirically (measured data). To this end, we used the discrete Fourier transform (DFT) ${ }^{3}$ to compute the Fourier transform of the empirical autocorrelation. Figs. 10(a) to 10(c) show some sample plots comparing the experimental and theoretical power spectrum for different values of the fading parameters. Again, an excellent fit can observed.

\footnotetext{
${ }^{3}$ The DFT was implemented by the FFT (Fast Fourier transform) algorithm.
} 


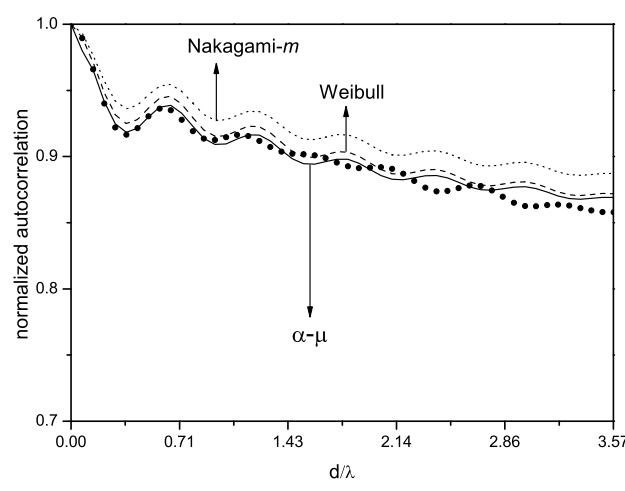

(a) Indoor measurement.

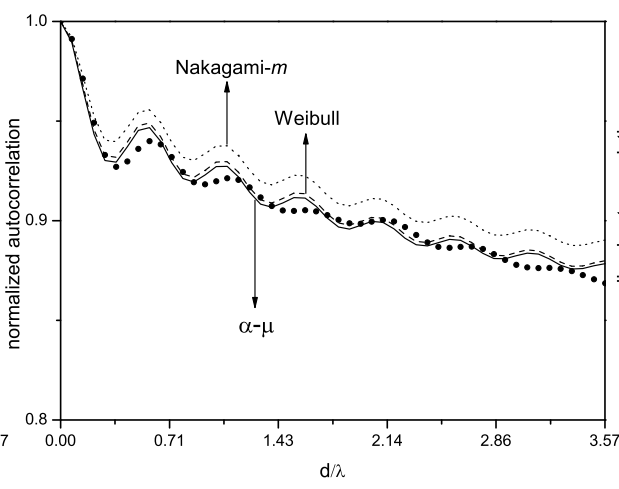

(b) Outdoor measurement.

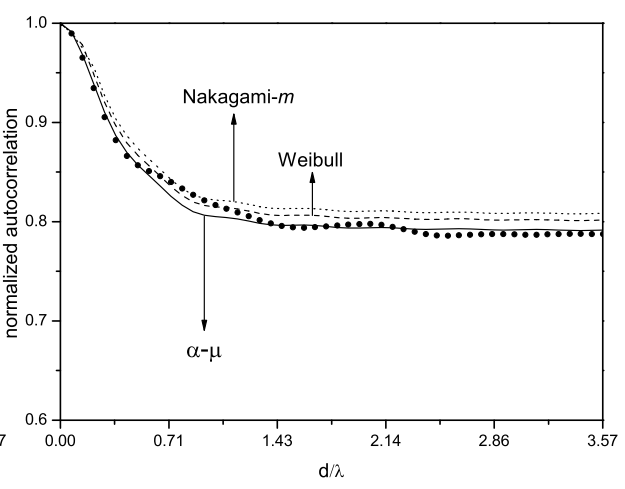

(c) Outdoor measurement.

Fig. 9. Empirical versus theoretical normalized autocorrelation functions (anisotropic scenarios).

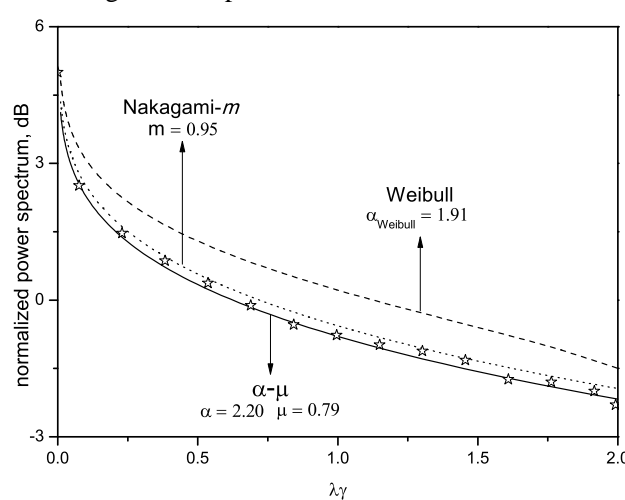

(a) Indoor measurement.

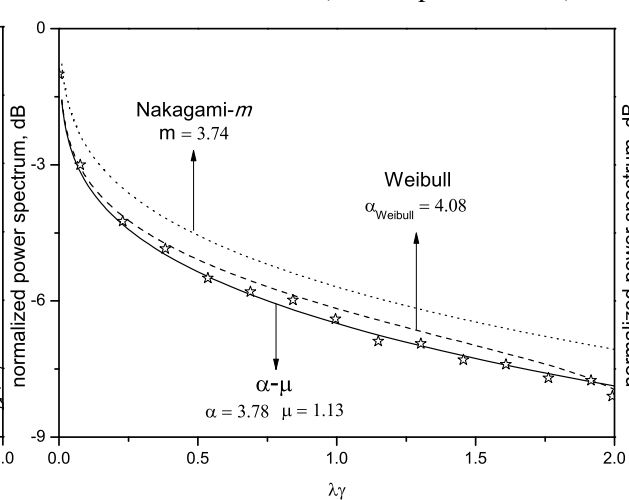

(b) Outdoor measurement.

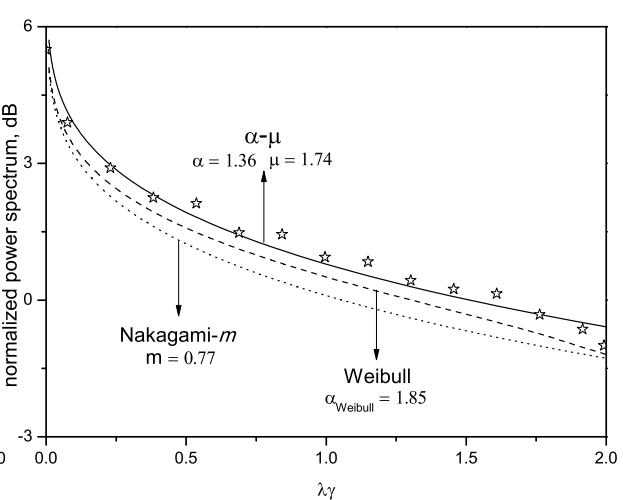

(c) Indoor measurement.

Fig. 10. Empirical versus theoretical normalized power spectrum functions (isotropic scenarios).

TABLE I

ESTIMATED VALUES OF THE FADING PARAMETERS AND ANISOTROPIC MODEL.

\begin{tabular}{|c|c|c|c|c|c|c|c|c|c|c|c|c|}
\hline Fig. & $\begin{array}{l}\text { Approximate orientation } \\
\text { to transmitter }\end{array}$ & $\hat{\alpha}$ & $\hat{\mu}$ & $\hat{m}$ & $\hat{\alpha}_{W \text { eibull }}$ & $\hat{\zeta}$ & $\hat{k}$ & $\hat{\varphi}$ & $\min (\mathrm{MSE})$ & $\begin{array}{c}\epsilon_{\alpha-\mu} \\
(\%)\end{array}$ & $\begin{array}{c}\epsilon_{\alpha} \\
(\%)\end{array}$ & $\begin{array}{c}\epsilon_{m} \\
(\%)\end{array}$ \\
\hline 9(a) & Parallel & 2.39 & 0.73 & 1.04 & 1.98 & 0.8 & 9.7 & 0 & $8.1 \mathrm{E}-4$ & 0.29 & 0.33 & 1.55 \\
\hline 9(b) & Parallel & 2.75 & 0.83 & 1.53 & 2.45 & 0.8 & 10 & 0 & $7.6 \mathrm{E}-4$ & 0.40 & 0.45 & 1.30 \\
\hline $9(\mathrm{c})$ & Perpendicular & 1.99 & 1.03 & 1.02 & 2.02 & 0.9 & 4.9 & 45 & $7.8 \mathrm{E}-4$ & 0.58 & 0.96 & 1.17 \\
\hline
\end{tabular}

\section{CONCLUSIONS}

In this paper, we reported the results of field trials aimed at investigating the second-order statistics of the $\alpha$ - $\mu$ fading signal, namely the autocorrelation function and envelope power spectrum. An excellent agreement between the experimental and the theoretical data was found. The measurements validate the autocorrelation formula derived in an exact manner in [4] for the $\alpha-\mu$ fading signal. Moreover, an accurate closed-form approximation to the power spectrum of the $\alpha-\mu$ envelope was also obtained and validated.

\section{REFERENCES}

[1] H. Suzuki, "A Statistical Model for Urban Radio Propagation," IEEE Trans. Commun., vol. 25, Jul. 1977.

[2] M. D. Yacoub, "Fading distributions and Co-Channel Interference in Wireless Systems," IEEE Antennas and Propagat. Mag., vol. 42, pp. 150-160, Feb. 2000.
[3] U. S. Dias, M. D. Yacoub, G. Fraidenraich, D. B. da Costa, and J. C. S. Santos Filho, "On the Weibull Autocorrelation and Power Spectrum Functions: Field Trials and Validation," IEEE Commun. Lett., vol. 10, pp. 710-712, Oct. 2006.

[4] M. D. Yacoub, "The $\alpha-\mu$ Distribution: A Physical Fading Model for the Stacy Distribution," IEEE Trans. Veh. Technol., vol. 56, no. 1, pp. 27-34, Jan. 2007.

[5] U. S. Dias, M. D. Yacoub, J. C. S. Santos Filho, G. Fraidenraich, and D. B. da Costa, "On the Nakagami-m Autocorrelation and Power Spectrum Functions: Field Trials and Validation," in IEEE International Telecommunications Symposium, ITS'06, Fortaleza-CE, Brazil, Sep. 2006, pp. 253-256.

[6] H. Hashemi, "The indoor radio propagation channel," Proc. IEEE, vol. 81, pp. 943-968, Jul. 1993.

[7] G. Tzeremes and C. G. Christodoulou, "Use of Weibull distribution for describing outdoor multipath fading," in Proc. IEEE Antennas and Propagation Soc. Int. Symp., vol. 1, Jun. 2002, pp. 232-235.

[8] F. Babich and G. Lombardi, "Statistical analysis and characterization of the indoor propagation channel," IEEE Trans. Commun., vol. 48, pp. 455-464, Mar. 2000.

[9] W. C. Y. Lee, "Finding the approximate angular probability density function of wave arrival by using a directional antenna," IEEE Trans. Antennas Propagat., vol. 21, pp. 328-334, 1973.

[10] M. Abramowitz and I. A. Stegun, Handbook of Mathematical Functions 
XXVII SIMPÓSIO BRASILEIRO DE TELECOMUNICAÇÕES - SBrT 2009, DE 29 DE SETEMBRO A 2 DE OUTUBRO DE 2009, BLUMENAU, SC with Formulas, Graphs, and Mathematical Tables. New York: Dover, 1972.

[11] W. C. Jakes, Microwave Mobile Communications. New York, USA: Wiley, 1974.

[12] R. Von Mises, "Über die 'Ganzzahligkeit' der Atomgewicht und verwandte Fragen," Phys. Z., vol. 19, pp. 490-500, 1918.

[13] I. S. Gradshteyn and I. M. Ryzhik, Table of Integrals, Series, and Products, 6th ed. Academic Press, 2000.

[14] R. Gonin and A. H. Money, Nonlinear $L_{p}$-Norm Estimation. New York: Marcel-Dekker, 1989. 